\title{
Research on the Protection and Utilization of Tea Tree Resources in Chaozhou -- Phoenix "Old Bundled Narcissus" is taken as an Example
}

\author{
Enyu Shi \\ Hanshan Normal University, Chaozhou Guangdong, 521000, China
}

Keywords: Chaozhou tea, Tea tree resources, Protection, Utilization.

\begin{abstract}
Chaozhou is a major city of tea culture and has a close relationship with tea. According to statistics, there are more than 3,000 tea trees in Chaozhou over 200 years old and more than 10,000 tea trees over 100 years old. Most of these ancient teas are native narcissus tea varieties. Narcissus tea is a new species formed by a local variety of red pungent tea that naturally mutates, and its quality is not high. In recent years, with people's enthusiasm for high-grade single-cone tea, narcissus tea has been slowly neglected. Many old bundled tea trees have been replaced with new single bundled cultivars. This paper analyzes the composition of tea quality, and urges people to pay attention and protect the old bundled tea trees, so as to rejuvenate the ancient tea trees.
\end{abstract}

\section{Introduction}

The tea production in the Fenghuang Mountain in Chaozhou has a long history, which, according to statistics, can be traced back to the Tang Dynasty. The folks are rumored that the Song Emperor Zhao Bing passed by the Phoenix Mountain when he fled southward, thirsty. His attendants picked a kind of leaf with a tip resemblong a jiao beak from the hill and made it into tea. Zhao Bing felt comfortable after drinking it. Therefore, later generations widely planted the tea trees, which were called the "song type" or the "jiao beak tea". As the Guangdong General Annals Manuscript in the Jiajing Period of the Ming Dynasty recorded, "The tea in Chaoan is the best." This shows that Chaozhou had become an important tea production area in Guangdong by that time. Phoenix tea was gradually known by people in the Qing Dynasty. Since it hit acroos Panama, it was included in the national tea.

Since the founding of New China, the tea production in Chaozhou has entered a new stage of rapid development. Since 1982, Phoenix tea has been rated as national tea for many times. Since 1990, the area of tea gardens in Chaozhou tea growing areas has continuously increased, and the quality of tea has also greatly improved. After the 1970s, the Phoenix Mountain planted white leaf single-bundled tea on a large scale. Therefore, most of the existing white leaf single- bundled tea trees are mostly 30-40 years old, which are not regarded as old bundles yet. Thus, when we talk about the old-bundled tea, we refer to the 50-year-old phoenix narcissus and orchid tea. Old single-bundled tea refers to narcissus and a small number of famous bundles. To some extent, "Phoenix Narcissus" is synonymous with old-bundled tea.

\section{The Quality and Characteristics of "Old-bundled Narcissus"}

Phoenix single plexus tea is an oolong tea. The shape of the bar is stout, even and straight, the color is brown, oily and bright, and has red spots of cinnabar; the brewing fragrance lasts, there is a unique natural flower fragrance, the taste is strong and fresh and cool, the throat is sweet, and it has a unique Mountain rhyme character. Phoenix Single Cong tea was selected from the Phoenix daffodil group for the selection of excellent individual tea plants, which were cultivated, picked, and processed according to different processing methods. Phoenix Single Cong tea has a long-lasting brewing fragrance, a unique natural floral scent, a strong mellow taste, a moist throat, and a unique tea product. 


\subsection{The Processing Method of "Old-bundled Narcissus"}

Phoenix Daffodils is the mother of single plexus tea. Single plexus tea is plucked, and when the new tea buds germinate to a small opening (i.e., budding), they are picked on the basis of a bud two or three leaf standard, and are picked up by the "riding and picking tea" technique, and placed gently on the tea to spare. When picking tea, you must follow the principle of "not to adopt when there is strong sunlight, not to collect in rainy weather, and not to adopt in fog days". This is because it is a summary of experience to avoid these extreme weather conditions when making tea.

The best time for tea picking is usually afternoon, so when the sun is not too strong, there is plenty of time for drying, and processing at night. All the tea production was performed at night, mainly because the nighttime temperature is good for the fermentation of raw tea. Tea green leaves are dried, green, green, blue, rubbing, baking and other processing processes, which lasted for 10 hours to make a tea full of tea. The processing method of Phoenix single-bundled tea is a new one developed based on the method of picking and processing Phoenix narcissus.

\subsection{The Quality of "Old-bundled Narcissus"}

The "Old-bundled Narcissus" tea processed by this method is thick, well-rounded, brownish in color, lubricious and bright, and has red spots of cinnabar; the brewing fragrance lasts long and has a unique natural orchid fragrance. The taste is strong, mellow, fresh, cool, moist and sweet; the color of the tea soup is clear, bright and yellow, with the edge of the leaves red and the belly yellow. The method of making tea is also very particular, using a specially made Yixing small ceramic teapot and drinking it with a small white porcelain cup. The tea should be much, the water little and time short. At the first making its fragrance is smelled; at the second making the taste is enjoyed; at the third making the soup is drunk. It's easy for to get released and calm after drinking.

With long tea trees, deep deposits, clear clumps, aromas, indulgence, long-lasting aromas, dark red and bright soup packets, "Old-bundled Narcissus" possesses the characteristics of high-grade Phoenix tea. Due to the high content of polyphenols in the "old-bundled Narcissus" tea, it is endowed with rich endoplasmic potency and strong intensity, which is really appreciated by those who really understand tea.

\section{The Dilemma of "Old-bundled Narcissus"}

Phoenix narcissus is native to the Phoenix Mountain in Chaozhou, Guangdong, and is a new natural variation of wild red tea. Due to the natural formation plus the earlier crude processing method, the narcissus tea does not have the characteristics of the natural floral fragrance of the single-bundled tea today; the color of the tea soup is not as clear and bright as single-bundled tea; the narcissus tea has a strong taste, and the leaf bottom is not even or balanced, without the green leaves and red edges of single-bundled tea. It is because of some shortcomings of narcissus tea that it is still regarded as inferior tea although "old-bundled narcissus" is the mother of the Phoenix single bundles.

\subsection{Seriously Damaged "Old-bundled Narcissus"}

With the rapid development of society, in recent years, people's living standards have greatly improved, and the demand for tea products has become higher and higher. In particular, the strong natural floral fragrance of single-bundled tea has been pursued. For instance, "Huangzhi" and "Yashi" incenses are popular. This also reflects the increasing trend of people's pursuit of fashion during the period of rapid social development. "Huangzhi" and "Yashi" incenses have become a kind of fashion because their rich aroma has satisfied people's curiosity to some extent. For a certain period of time, there is a situation where demand exceeds supply for this kind of fragrant tea, with skyrocketing prices.

\section{2 "Old-bundled Narcissus" Deviating from Market Demand}

Phoenix "old-bundled narcissus" is becoming rare at present. The reason why "old-bundled narcissus" is not valued by the tea growers is that its fragrance is not obvious despite its strong flavor, 
which does not suit the fickle mood of people today. Few people like " old-bundled narcissus", so that it gradually went out of the public eye. Only a few "old tea drinkers" is especially fond of "old-bundled narcissus".

\section{To Rejuvenate "Old-bundled Narcissus"}

"Phoenix narcissus" is named after the narcissus variety with the place. The "Phoenix Narcissus" produced in Phoenix Mountain in Chaozhou is a species of endemic breed that breeds sexually, which is the small tree type, middle leaf species, with a sparse, erect or semi-developed trunk. The tip of the phoenix narcissus has a distinct feature: the tip of the leaf is slightly curved, to the left or right, resembling a bird's beak, so it is called "bird's beak tea." Due to the unique clump flavor and rich endoplasm of narcissus tea, plus its high cost-effectiveness, "people who really understand tea" have a special liking for it. The rich "old charm" of narcissus makes people deeply appreciate the charm of tea culture.

Is "old-bundled narcissus" really old? Will it really go out of people's eye? The author's answer is negative, and the author also firmly believes that "old-bundled narcissus" will have a reappearance of glory, because it has a deeper understanding than the intrigues of fashion teas such as Yashi fragrance. Then, how can we make the hidden "old clumps of daffodils" to shine again?

\subsection{To Continuously enrich the cultural connotation of "Old-bundled Narcissus"}

"Old bundles" are a kind of historical accumulation, with a long history and deep charm. In order to elaborate this history to the people who love tea, it's best to carry out activities of popularizing tea culture, so that people can improve their tea tasting ability.

The Phoenix Narcissus single bundle has the reputation of "beautiful shape, green color, rich fragrant and sweet taste”. The tea bar is straight and hypertrophic, with a yellowish-brown color and a squid skin color that is oily and shiny. The tea soup is orange and clear; along the wall of the cup will be a golden halo; the bottom of leaves is soft, the edge is vermilion, the belly is yellow, the taste is mellow and sweet, with natural lasting flora fragrance, high foam resistance, loved by those who really understand tea. Why? Because of its high cost performance. Those who understand tea can buy high-quality tea products that reflect the cultural heritage of Chaozhou tea at a very low price.

Stories can arouse people's interest. The legend about Song tea is very attractive in itself. During the Southern Song Dynasty, the Southern Song Emperor fled south through the Phoenix Mountain and was thirsty. At this time, there were a bunch of tea twigs and pedicle tea fruit. The tea twigs helped the Song Emperor to relieve the thirst and the tea fruit bred into the "Song tea trees". " Because it is brought by a colorful phoenix, it is also known as "bird's beak tea." Although it was the last emperor of the Song Dynasty, it was probably the only emperor who visited Chaozhou after all. This anecdote may become a powerful promoter of "old-bundled narcissus".

\subsection{To Popularize tea culture knowledge and improve tea appreciation}

It is necessary to cultivate people's tea culture and improve their ability to evaluate tea products. It should be said that tea is now considered a fashion, nothing more. The pursuit of drinking tea as a fashion will inevitably bring about the pursuit of so-called "fashionable" tea products. The reason why we talk about "tea appreciation" is to taste the perceived cultural connotations through tea tasting. Without good cultural qualities, it is impossible to appreciate the deep and rich connotation of tea culture. Therefore, we must start by improving people's tea culture and improve people's ability to evaluate tea products. Only in this way can we remove people's shallow fashion-seeking behavior and turn to improve quality and richness. This may re-enter people's horizons for "old-bundled narcissus", which is hidden and deep in meaning.

"Old-bundled narcissus" tea is positioned as a kind of beverage, has the functions of thirst resistance, refreshing and digestion, and has become the daily necessities for "opening seven things every day, daily necessities, vinegar, salt and vinegar tea", then as a necessity of life, Phoenix "old-bundled narcissus" embodies only its material properties. As a material property satisfies the 
needs of people's lives, tea plays the material function of tea, and reflects its material properties. Then "old clumps of narcissus" tea can only be a low-value tea. In addition to the physical properties of tea, there is a more important, higher level of function, that is, cultural functions. When we are tasting tea, we often say that the taste of tea is very good. This reflects the people's love and pursuit of the tea's charm, and it belongs to the spiritual enjoyment. What is embodied is the cultural properties of tea. The "old-bundled narcissus" can only truly reflect their own values and become the objects that people compete to acquire when they are only able to grow up to the cultural attributes of tea.

\subsection{To Guide people's consumption}

Jobs believes that consumption can be guided. This view overthrows the traditional concept of "the customer is God." In fact, the vast majority of consumers have a very limited understanding of tea. Therefore, the pursuit of fashion must exist. "Old-bundled narcissus" should become the spiritual level of enjoyment that people pursue. This spiritual enjoyment naturally transcends the physical properties of tea as a beverage and rises to the spiritual level. As long as the "old-bundled narcissus" has specific cultural attributes, then it should be the goal of people's pursuit of spiritual enjoyment. As long as "old-bundled narcissus" has specific cultural attributes, they should become the goal of spiritual enjoyment pursued by people. Its value will naturally go up. To attract consumers through the promotion of tea culture and the power of cultural marketing is a very effective way to guide consumer behavior. It is worthy of our thinking and exploration.

\section{Summary}

The rise from "drinking" tea to "appreciating" tea shows that Gongfu tea has exceeded the material properties of tea and has entered a higher level of cultural attributes. The special cultural attributes of Chaozhou Gongfu tea have a higher level of demand for tea quality. This requires that the development and promotion of tea culture lies in the improvement of tea quality and cultural attributes. Because from the perspective of economics, the value of the cultural attribute of goods is much more important than the value of the physical attributes of the goods. The material properties of tea products are marketed as essential products for tea. They embody that tea itself has the functions of thirst quenching, digestion, etc., and has the material value of food. The tea with cultural properties can make people feel good and have spiritual enjoyment. The value of a cultural property far exceeds the value of its material properties and can be said to be priceless. Well, the "old-bundled narcissus", which seems to be "outdated" in the tea industry, must revive its glory and must start with culture. The key to improving the competitiveness of "old-bundled narcissus" is how to establish their cultural concepts and enhance the value of their cultural attributes. The "old-bundled narcissus" must continue to innovate, must vigorously carry forward, continue to enrich the connotation of their culture, and constantly improve the cultural attributes of tea products. Only in this way can the “old-bundled narcissus” effectively regain their glory.

\section{Acknowledgement}

This research was financially supported by the achievements of the "2016 Chaozhou Culture Research Fund” of Chaozhou Municipal Party Committee Propaganda Department---Inheritance, Development, and Innovation Development of Chaozhou Tea Culture.

\section{References}

[1] Chief Editing by Yu Yue, Chinese Tea Culture Series Guangming Daily Press 1999.

[2] By Huang Ruiguang, Phoenix Single Bundles, China Agriculture Press, 2006.

[3] Chief Editing by Chen Xiangjun, Introspection of Non-material Cultural Heritage of Chaozhou, Chinese Literature and History Press, 2009. 
[4] By Qiu Taorui, Phoenix Tea in China - Tea History, Tea Stories and Tea People, Shenzhen Press Group Publishing House, 2015. 